\title{
Prediction of toxicities of prostate cancer radiotherapy
}

\author{
B. HOSTOVA ${ }^{1,2, *}$, P. MATULA ${ }^{1}$, P. DUBINSKY ${ }^{1}$ \\ ${ }^{1}$ East Slovakia Institute of Oncology, Rastislavova 43, 04191 Kosice, Slovak Republic; ${ }^{2}$ P. J. Safarik University, Faculty of Science, Srobarova 2, \\ 04154 Kosice, Slovak Republic \\ ${ }^{*}$ Correspondence: barbora.petruskova@student.upjs.sk
}

Received August 27, 2014 / Accepted September 7, 2015

\begin{abstract}
We treated a cohort of 116 patients with prostate cancer with three-dimensional conformal hypofractionated radiotherapy to a total dose of $52.8 \mathrm{~Gy}$ in 16 fractions (3.3 Gy per fraction). The correlation between acute and late gastrointestinal (GI) and genitourinary $(\mathrm{GU})$ toxicity and dose-volume parameters was analysed. Comparison of observed incidence of toxicity and normal tissue complication probability calculated from dose-volume histograms (DVH) of all patients using radiobiological Lyman-Kutcher-Burman model was performed. The results of our study suggest that acute gastrointestinal toxicity $\geq$ grade 2 $(\mathrm{G} 2)$ is the significant predictor of late toxicity $\geq \mathrm{G} 2(\mathrm{p}=0.006)$. Observed incidence of acute and late GI toxicities $\geq \mathrm{G} 2$ was 9.7\% and $11.5 \%$, respectively. NTCPs of acute and late GI complications $\geq$ G2 were $11.3 \%$ and $2.5 \%$. Observed incidence of late GU toxicity $\geq$ G2 was $14.2 \%$, NTCP was $0.8 \%$. Comparison of calculated probability of acute GI toxicity $\geq$ G2 and observed incidence indicates that parameters of radiobiological models are set appropriately. Comparison of observed incidence of late GI and GU complications $\geq$ G2 and calculated NTCPs shows the need of refinement of LKB model parameters for acute and late GI and GU complications $\geq \mathrm{G} 2$.
\end{abstract}

Key words: prostate cancer, radiotherapy, acute and late toxicity, radiobiological modeling

Radiotherapy is one of modalities in treatment of prostate cancer. Common side effects of radiotherapy include early and late genitourinary and gastrointestinal toxicity, both limiting factors of dose escalation. Acute rectal toxicity might be a predictor of late rectal toxicity and can be severe enough to interrupt the planned treatment course. A number of studies have proved close correlation between acute and late rectal toxicity and a number of dosimetric parameters $[1,2]$.

Treatment planning systems (TPS) allow to calculate 3D distributions of absorbed dose but do not provide information about response of tumor and healthy tissues after irradiation which depends on several factors. Radiobiological models biological effective dose (BED) and normal tissue complication probability (NTCP) provide information about biological response of normal tissue.

The aim of this study is to report observed acute and late GU and GI toxicity after hypofractionated radiotherapy in relation to dose-volume parameters. Moreover, we compare observed incidence of late GU and acute and late GI toxicity with results of radiobiological modeling.

\section{Patients and methods}

A cohort of 116 consecutive patients with stage T1a-T3 prostate cancer treated from March 2007 to February 2009 in East Slovakia Institute of Oncology in Kosice, Slovak Republic, were enrolled to our analysis. Median age of patients was 69 years. Risk categories were defined as reported by the National Comprehensive Cancer Network (NCCN) clinical practice guidelines in oncology [3]. Main patient characteristics are summarized in Table 1.

Radiotherapy. The gross tumor volume (GTV), clinical target volume (CTV) and planning treatment volume (PTV) were delineated on computerized tomography $(\mathrm{CT})$ images by a radiation oncologist. GTV was expanded by $3 \mathrm{~mm}$ to CTV in intermediate and high risk patients. The PTV was created adding $1 \mathrm{~cm}$ in each direction except posteriorly, were the 7 $\mathrm{mm}$ margin was added. The organ at risk delineation included rectum and bladder. Entire rectum was manually contoured from the level of anus to the sigmoid loop. The PTV was covered using a four-field box three-dimensional conformal technique (3D CRT). The prescribed dose was 52.8 Gy in 16 
daily fractions of $3.3 \mathrm{~Gy}$. Calculated equivalent total dose at 2 Gy fractionation of this schedule is 72.4 Gy assuming $\alpha / \beta$ ratio of $1.5 \mathrm{~Gy}$.

Irradiation was delivered by $18 \mathrm{MeV}$ photons of linear accelerator with multileaf collimator. The patient position was verified by weekly portal imaging.

Correlation between acute and late toxicity. GI and GU toxicity was classified according to the Common Toxicity Criteria scoring v. 3 [4]. We examined observed acute GI/GU toxicity $\geq \mathrm{G} 2$ as the predictive factor for late GI/GU toxicity $\geq \mathrm{G} 2$.

DVH analysis and dosimetric parameters. $\mathrm{D}_{\max }$ (maximal dose) and $\mathrm{D}_{\text {mean }}$ (mean dose) in rectum and bladder obtained from DVH were evaluated as potential predictors for acute and late GI and GU toxicity $\geq G 2$. $D_{\text {max }}$ and $D_{\text {mean }}$ were assessed from $\mathrm{DVH}$ for each patient individually. The correlations between dose-volume parameters from study Arcangeli et al. [1] and Marzi et al. [5]: V46 < 30\% (less than 30\% of rectal volume receive dose higher than $46 \mathrm{~Gy}), V 33<50 \%$ and parameter from our previous experience: $V 50<25 \%$ and acute and late GI toxicity $\geq$ G2 were assessed by Pearson correlation coefficient (r). Statistical analysis was performed using SPSS Statistics software.

Radiobiological modeling. Cumulative dose-volume histograms (DVH) were created by treatment planning in TPS Plato. The Lyman-Kutcher-Burman model (LKB) described in

Table 1. Patient characteristics

\begin{tabular}{|c|c|}
\hline Characteristic & No. of patients (\%) \\
\hline Age, median (range) & $69(50-88)$ year \\
\hline \multicolumn{2}{|l|}{ Stage } \\
\hline T1a b, c & $28(24.3 \%)$ \\
\hline $\mathrm{T} 2 \mathrm{a}$ & $11(9.6 \%)$ \\
\hline $\mathrm{T} 2 \mathrm{~b}$ & $33(28.7 \%)$ \\
\hline $\mathrm{T} 2 \mathrm{c}$ & $13(11.3 \%)$ \\
\hline $\mathrm{T} 3$ & $30(26.1 \%)$ \\
\hline \multicolumn{2}{|l|}{ Gleason score } \\
\hline $2-6$ & $73(64.6 \%)$ \\
\hline 7 & $27(23.9 \%)$ \\
\hline $8-10$ & $13(11.5 \%)$ \\
\hline \multicolumn{2}{|l|}{ PSA } \\
\hline$<10 \mathrm{ng} / \mathrm{ml}$ & $47(40.5 \%)$ \\
\hline $10-20 \mathrm{ng} / \mathrm{ml}$ & $32(27.6 \%)$ \\
\hline$>20 \mathrm{ng} / \mathrm{ml}$ & $37(31.9 \%)$ \\
\hline \multicolumn{2}{|l|}{ Risk category } \\
\hline LR & $18(15.5 \%)$ \\
\hline IR & $41(35.3 \%)$ \\
\hline HR & $57(49.1 \%)$ \\
\hline \multicolumn{2}{|l|}{ Hormonal therapy } \\
\hline Yes & $98(86.7 \%)$ \\
\hline No & $15(13.3 \%)$ \\
\hline
\end{tabular}

PSA - prostatic specific antigen; LR - low risk; IR - intermediate risk; HR, high risk
Appendix (A.1) was used to predict acute and late GI toxicity $\geq \mathrm{G} 2$ and the acute $\mathrm{GU}$ toxicity $\geq \mathrm{G} 2$.

The model parameters $n=0.08, m=0.17, T D_{50}=53$ Gy were involved in the calculations of NTCP for acute GI toxicity $\geq$ G2 [6]. The prediction of acute reactions in rectum was based on study Fowler et al. [7], where the extrapolation from oral or pharyngeal mucosa to rectal mucosa was done. In BED was included cell proliferation (A.3) with parameters: $\alpha=0.35 \mathrm{~Gy}^{-1}$, $\alpha / \beta=10 \mathrm{~Gy}, T_{k}=7$ days and $T_{p}=2.5$ days [7]. The acute G2 GU toxicity was not calculated because of the lack of radiobiological characteristic in published studies.

The model parameters $\alpha / \beta=3 G y, n=0.09, m=0.13$, $T D_{50}=76.8 \mathrm{~Gy}$ and $\alpha / \beta=3.4 \mathrm{~Gy}, n=0.13, m=0.11, T D_{50}=79.3 \mathrm{~Gy}$ were used for NTCP calculations for late GI and GU toxicity $\geq$ G2, respectively [8]. NTCPs were calculated with the use of $\operatorname{BED}($ A.2).

Radiobiological calculations were performed using BioGray software and homemade programs in MS Excel and ROOT. Software BioGray was developed in our department as evaluating tool for treatment plans from radiobiological point of view [9].

\section{Results}

Incidence of GU and GI toxicity. The median follow-up was 60 months (range 20 to 78 months). Results of observed incidence of acute and late GI and GU toxicity are summarized in the Table 2 .

Correlation between acute and late toxicity. A significant correlation was found between acute and late GI toxicity $\geq$ G2 $(r=0.256, p=0.006)$. A non-significant correlation was found between acute GU toxicity and late GU toxicity $\geq \mathrm{G} 2$ $(\mathrm{r}=0.092, \mathrm{p}=0.331)$.

DVH analysis and dosimetric parameters. No significant correlation was observed between $\mathrm{D}_{\text {mean }}, \mathrm{D}_{\text {max }}$ in rectum/bladder and acute and late GI/GU toxicity $\geq$ G2. No significant correlation was found between dose-volume parameters V50, V45 and V33 in rectum and acute or late GI toxicity $\geq$ G2. The results of univariate analysis of potential predictors for acute and late GI and GU toxicity $\geq$ G2 are summarized in Table 3.

Table 2. Acute and late GU/GI toxicity

\begin{tabular}{cccc}
\hline Acute GU toxicity & \multicolumn{3}{c}{ Acute GI toxicity } \\
\hline G0 & $4,4 \%$ & $\mathrm{G} 0$ & $3,5 \%$ \\
G1 & $83,2 \%$ & $\mathrm{G} 1$ & $86,7 \%$ \\
G2 & $11,5 \%$ & $\mathrm{G} 2$ & $9,7 \%$ \\
G3 & $0,9 \%$ & $\mathrm{G} 3$ & $0,0 \%$ \\
\hline Late GU toxicity & \multicolumn{3}{c}{ Late GI toxicity } \\
\hline G0 & $45,1 \%$ & $\mathrm{G} 0$ & $73,5 \%$ \\
G1 & $40,7 \%$ & $\mathrm{G} 1$ & $15,0 \%$ \\
G3 & $9,7 \%$ & $\mathrm{G} 2$ & $9,7 \%$ \\
\hline
\end{tabular}

G- Grade 
Radiobiological modeling. We calculated average NTCP of acute and late G2 GI and GU toxicity $\geq$ G2 with standard deviation. Predicted average acute GI toxicity $\geq$ G2 with standard deviation was $(11.3 \pm 3.0) \%$. Average NTCPs of late GI and GU toxicities $\geq \mathrm{G} 2$ were $(2.5 \pm 1.1) \%$ and $(0.8 \pm 0.8) \%$. The comparison of observed incidence of GI and GU toxicities $\geq \mathrm{G} 2$ with results of radiobiological modeling is summarized in Table 4. Results of radiobiological modeling of acute GI toxicity $\geq \mathrm{G} 2$ are close to incidence of observed acute rectal toxicity. This indicates that parameters of LKB model and $\mathrm{BED}$ for acute GI toxicity $\geq \mathrm{G} 2$ are set appropriately. Clinical outcome for GI and GU late toxicities $\geq$ G2 was outside the range of calculated NTCP. This shows a need to further specify LKB parameters for late GI and GU toxicity $\geq$ G2.

\section{Discussion}

Study included 116 patients with prostate cancer followed for 60 months. Observed incidence of late GI toxicity $\geq$ G2 was $11.5 \%$ This was low compared to results reported by Arcangeli et al. [1] (incidence of late GI toxicity $\geq$ G2 was 17\%) and close to results published by Marzi et al. [5] (incidence of late rectal toxicity $\geq \mathrm{G} 2$ was $12.3 \%$ ) where authors used total dose of 62 Gy in 20 fractions. Comparison of toxicity between studies is difficult due to low sensitivity of subjective toxicity grading.

Correlation between acute and late toxicity. Results of our study showed that acute GI toxicity $\geq$ G2 is predictor for late GI toxicity $\geq \mathrm{G} 2$. Acute GI toxicity as a predictive factor

Table 3. Results of univariate analysis of potential predictors for acute/ late GU/GI toxicity $\geq$ G2

\begin{tabular}{|c|c|c|c|c|}
\hline & $\begin{array}{l}\text { acute GI } \\
\text { toxicity }\end{array}$ & $\begin{array}{l}\text { late GI } \\
\text { toxicity }\end{array}$ & $\begin{array}{l}\text { acute } \mathrm{GU} \\
\text { toxicity }\end{array}$ & $\begin{array}{l}\text { late } \mathrm{GU} \\
\text { toxicity }\end{array}$ \\
\hline \multicolumn{5}{|c|}{$\mathrm{D}_{\max }$ in rectum } \\
\hline $\mathrm{r}$ & 0.048 & 0.083 & & \\
\hline $\mathrm{p}$-value & 0.614 & 0.384 & & \\
\hline \multicolumn{5}{|c|}{$\mathrm{D}_{\text {mean }}$ in rectum } \\
\hline $\mathrm{r}$ & 0.043 & 0.106 & & \\
\hline $\mathrm{p}$-value & 0.650 & 0.265 & & \\
\hline \multicolumn{5}{|c|}{$\mathrm{D}_{\max }$ in bladder } \\
\hline $\mathrm{r}$ & & & -0.023 & -0.113 \\
\hline $\mathrm{p}$-value & & & 0.807 & 0.233 \\
\hline \multicolumn{5}{|c|}{$\mathrm{D}_{\text {mean }}$ in bladder } \\
\hline $\mathrm{r}$ & & & -0.071 & 0.140 \\
\hline p-value & & & 0.453 & 0.139 \\
\hline \multicolumn{5}{|l|}{ V50 } \\
\hline $\mathrm{r}$ & 0.142 & 0.117 & & \\
\hline p-value & 0.133 & 0.218 & & \\
\hline \multicolumn{5}{|l|}{ V45 } \\
\hline $\mathrm{r}$ & -0.156 & -0.079 & & \\
\hline $\mathrm{p}$-value & 0.099 & 0.404 & & \\
\hline \multicolumn{5}{|l|}{ V33 } \\
\hline $\mathrm{r}$ & -0.020 & 0.081 & & \\
\hline $\mathrm{p}$-value & 0.832 & 0.393 & & \\
\hline
\end{tabular}

$\mathrm{r}$ - Pearson correlation coefficient for late GI toxicity was identified in other studies, Arcangeli et al. [1] and Jereczek-Fossa et al. [2].

Acute GU toxicity $\geq \mathrm{G} 2$ as a predictor for late GU toxicity $\geq \mathrm{G} 2$ was not confirmed by our analysis. In contrary to our analysis, other studies [1], [2], [14] found significant correlation between acute and late urinary side effects. Anyhow the analysis of radiation induced toxicity in bladder is difficult because of pre-existing dysfunction correlated with age and previous surgery [2]. Authors of several studies [10-12] suggested that late urinary toxicity is mainly influenced by the presence of urinary symptoms before the start of radiotherapy. Some of these pre-existing symptoms might have been erroneously registered as acute or even late toxicity [2].

DVH analysis and dosimetric parameters. A number of studies have analyzed dose-volume parameters in order to find any predictive factor of acute or late radiation induced rectal toxicity. These studies provide different results. Variability of dose-volume constraints is associated with different RT technique, volume definition, total dose and fractionation, choice of endpoint and method of scoring toxicity [15]. Two dose-volume constraints for rectum used in our analysis originated from study Marzi et al. [5] and Arcangeli et al. [1] based on authors previous experiences that $87.5 \%$ and $62.5 \%$ of prescribed dose should be $<30 \%$ and $<50 \%$ of rectal wall, respectively. Our analysis did not show any of these dose-volume parameters as predictive factors for acute or late gastrointestinal toxicity $\geq$ G2. The reason may be different scoring of toxicity, Radiation Therapy Oncology Group/European Organization for Research and Treatment of Cancer system (RTOG/EORTC) [16] vs. NCCN [3] and different definition of organ at risk (rectal wall vs. rectum).

Dose-volume parameter for urinary toxicity were not examined because of variable volume of bladder during treatment course It cannot be expected that DVH of bladder obtained from single computed tomography (CT) imaging represents the true dose distribution delivered to the bladder during treatment course [17].

Table 4. Comparison of observed incidence of GI/GU toxicity $\geq$ G2 and results of radiobiological modeling with the use of LKB model

\begin{tabular}{ccc}
\hline & $\begin{array}{c}\text { Observed } \\
\text { incidence }\end{array}$ & $\begin{array}{c}\text { Mean NTCP } \\
\text { with SD }\end{array}$ \\
\hline GI toxicity $\geq G 2$ & & \\
Acute & $9.7 \%$ & $(11.3 \pm 3.0) \%$ \\
Late & $11.5 \%$ & $(2.5 \pm 1.1) \%$ \\
GU toxicity $\geq G 2$ & & $(0.8 \pm 0.8) \%$ \\
Late & $14.2 \%$ &
\end{tabular}




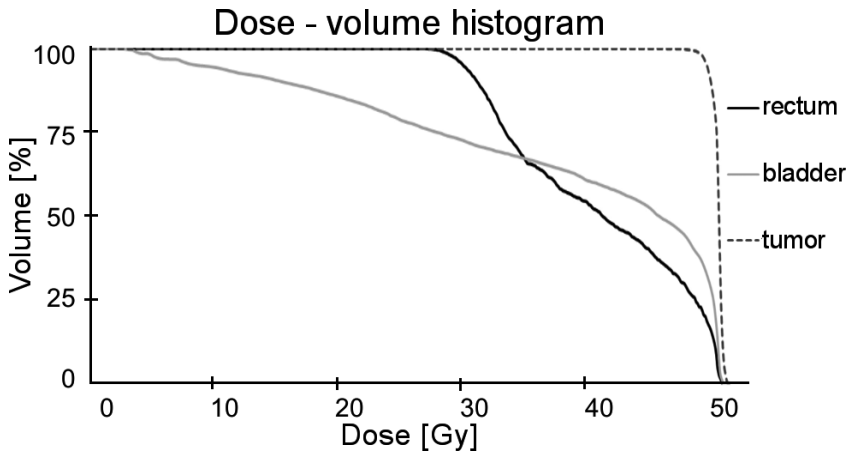

Figure 1. Dose-volume histogram of prostate (dashed line), rectum (black line) and bladder (grey line)

Radiobiological modeling. Normal tissue complication probability was calculated for acute and late effects of radiotherapy on rectum and late effects on bladder. From perspective of parameters needed for NTCP calculations, rectum is better described than bladder in literature, but there is a lack of radiobiological information about acute rectal toxicity. Strigari et al. [6] analyzed acute rectal toxicity and gained parameters of LKB model, which were used in our analysis. Fowler et al. [7] pointed out the possibility of using parameters of oral and pharyngeal mucosa. Based on this study, we found relation between observed incidence of acute rectal toxicity $\geq \mathrm{G} 2$ and calculated complication probability with use of LKB model what confirms suitability of this Fowler approach. Several studies were dedicated to late rectal complications from the view of radiobiological modeling $[5,18,19]$. In our radiobiological calculations of late rectal toxicity we used parameters from study Michalski et al. [8]. We noticed major difference between mean value of NTCP of late rectal toxicity $\geq \mathrm{G} 2$ and its observed incidence (see in Tab. 3). Parameters of LKB model used in our calculations do not describe our data very well. The aim of our following work will be to refine parameters of LKB model for late rectal toxicity.

Similarly, observed incidence of late urinary toxicity $\geq$ G2 was different to the results of radiobiological modeling. Observed incidence of late urinary toxicity might have been confounded by pre-existing urinary condition. Aim of our following work will be to refine parameters of LKB model also for late urinary complications.

Parameters of LKB and their confidence intervals will be estimated with the use of maximum likelihood analysis described in studies Marzi et al. [5], Rancati et al. [19] and Luijk et al. [20], respectively.

Our analysis did not confirm any significant correlations between DVH limits (Dmax, Dmean, VGy) and frequency of acute and late rectal and urinary toxicity. A substantial contribution of our work is a utilization of Fowler model for calculation acute NTCP of rectal mucosa toxicity after prostate cancer radiotherapy [7] and its comparison with

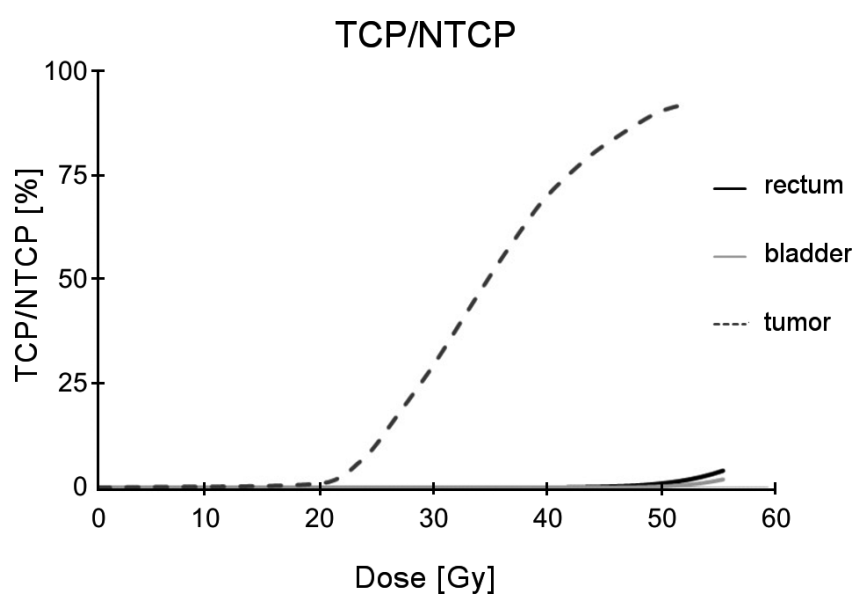

Figure 2. TCP (Tumor Control Probability) curve for prostate carcinoma (dashed line) and NTCP curves for rectum (black line) and bladder (grey line)

clinical observation for our hypofractionated regime $52.8 \mathrm{~Gy}$ in 16 fractions. The second substantial contribution of the work is the confirmation of significant correlation between acute and late rectal toxicity $\geq$ G2 after radiotherapy for prostate cancer.

$(\mathrm{p}=0,003)$

Appendix. Input for NTCP calculations is DVH (Figure 1). The four parameters of Lyman-Kutcher-Burman model can be calculated by

$$
N T C P=\frac{1}{\sqrt{2 \pi}} \int_{-\infty}^{t} e^{-\frac{x^{2}}{2}} d x
$$

where $t$ is defined as

$$
t=\frac{D-T D_{50}(v)}{m \cdot T D_{50}(v)}
$$

and $T D_{50}(v)=T D_{50}(1) \cdot v^{-n}(21)$. Parameter $n$ determines the volume dependence of NTCP, parameter $m$ determines the slope of NTCP curve and $T D_{50}$ is tolerance dose to the whole organ leading to $50 \%$ probability of complications. $D$ is total physical dose with which is patient irradiated and $v$ is a uniformly irradiated fraction of an organ.

$T D_{50}$ and $D$ in A.2 can be replaced by biological effective dose $B E D$,

$$
t=\frac{B E D-B E D_{50}(v)}{m \cdot B E D_{50}(v)}
$$

where $B E D$ is defined as

$$
B E D=n D\left(1+\frac{d}{\alpha / \beta}\right)
$$


$n$ is number of fractions and $\alpha / \beta$ ratio characterizes the radiosensitivity of tissue.

Repopulation of rectal mucosa has to be considered in calculation of BED for acute GI toxicity,

$$
B E D=n D\left(1+\frac{d}{\alpha / \beta}\right)-\frac{\ln (2)}{\alpha T_{p o t}}\left(T-T_{k}\right)
$$

where $T_{p o t}$ is the average doubling time of rectal mucosa, $T_{k}$ is the onset time of mucosa repopulation and $T$ is overall treatment time in days [7].

LKB model uses uniform dose distributions. Non-uniform DVH has to be converted into equivalent one with uniform dose distribution using Kutcher-Burman reduction technique [23]. This reduction algorithm reduces DVH to an effective volume which receives the maximum dose of the original DVH:

$$
v_{e f f}=\sum_{i} v_{i}\left(\frac{D_{i}}{D_{\max }}\right)^{\frac{1}{n}}
$$

NTCP curves of bladder and rectum and TCP curve (Tumor Control Probability) of prostate carcinoma calculated with radiobiological models can be seen in the Figure 2 .

\section{References}

[1] ARCANGELI G, FOWLER J, GOMELLINI S, ARCANGELI $S$, SARACINO B et al. Acute and late toxicity in a randomized trial of conventional versus hypofractionated three- dimensional conformal radiotherapy for prostate cancer. Int. J. Radiation Oncology Biol. Phys. 2011; 79: 1013-1021. http:// dx.doi.org/10.1016/j.ijrobp.2009.12.045

[2] JERECZEK-FOSSA BA, ZERINI D, FODOR C, SANTORO L, SERAFINI $\mathrm{F}$ et al. Correlation between acute and late toxicity in 973 prostate cancer patients treated with threedimensional conformal external beam radiotherapy. Int. J. Radiation Oncology Biol. Phys. 2010; 78: 2 6-34.

[3] NATIONAL COMPRENHENSIVE CANCER NETWORK. Prostate cancer. NCCN clinical practice guidelines in oncology. J. Natl. Compr. Canc. Net. 2004; 2: 224-248.

[4] CACNERTHERAPY EVALUATION PROGRAM.COMMON TERMINOLOGY CRITERIA FOR ADVERSE EVENTS, VERSION 3.0. Washington DC: Division of Cancer Treatment and Diagnosis, National Cancer Institute, National Institute of Health, Department of Health and Human Services; 2006.

[5] MARZI S, ARCANGELI G, SARACINO B, PETRONGARI MG, BRUZZANITI V et al. Relationship between rectal wall dose-volume constraints and radiobiologic indices of toxicity for patients with prostate cancer. Int. J. Radiation Oncology Biol. Phys. 2007; 68: 41-49. http://dx.doi.org/10.1016/j. ijrobp.2006.12.003

[6] STRIGARI L, ARCANGELI G,ARCANGELI S, BENASSI M. Mathematical model for evaluating incidence of acute rectal toxicity during conventional or hypofractionated radiotherapy courses for prostate cancer. Int. J. Radiation Oncology Biol. Phys. 2009; 73: 1454-1460. http://dx.doi.org/10.1016/j. ijrobp.2008.07.024

[7] FOWLERJF. The radiobiology of prostate cancer including new aspects of fractionated radiotherapy. Acta Oncologica 2005; 44: 265-276. http://dx.doi.org/10.1080/02841860410002824

[8] MICHALSKI JM, GAY H, JACSKON A, TUCKER S, DEASY JO. Radiation dose- volume effects in radiation-induced rectal injury. Int. J. Radiation Oncology Biol. Phys. 2010; 76: S123S129. http://dx.doi.org/10.1016/j.ijrobp.2009.03.078

[9] MATULA P, KONCIK J, JASENCAK M. Simultaneous radiobiological prediction of TCP/NTCP based on DVH and QUANTEC project using SW BioGray-Plus. Radiotehr. Oncol. 2010; 96: S610.

[10] SCHULTHEISS TE, LEE WR, HUNT MA, HANLON AL, PETER RS et al. Late GI and GU complications in treatment of prostate cancer. Int. J. Radiation Oncology Biol. Phys. 1997; 37: 3-11. http://dx.doi.org/10.1016/S0360-3016(96)00468-3

[11] LIU M, PICKLES T, BERTHELET E, AGRANOVICH A, KWA W et al. Urinary incontinence in prostate cancer patients treated with external beam radiotherapy. Radiotehr. Oncol. 2005; 74: 197-201. http://dx.doi.org/10.1016/j. radonc.2004.09.016

[12] SANDHU AS, ZELEFSKY MJ, LEE HJ, LOMBARDI D, FUKS $\mathrm{Z}$ et al. Long-term urinary toxicity after 3-dimensional conformal radiotherapy for prostate cancer in patients with prior history of transurethral resection. Int. J. Radiation Oncology Biol. Phys. 2000; 48: 643-647. http://dx.doi.org/10.1016/ $\underline{\mathrm{S} 0360-3016(00) 00714-8}$

[13] MORRIS D, EMAMI B, MAUCH PM, KONSKI AA, TAO ML et al. Evidence-based review of three-dimensional conformal radiotherapy for localized prostate cancer: an ASTRO outcomes initiative. Int. J. Radiation Oncology Biol. Phys. 2005; 62: 3-19. http://dx.doi.org/10.1016/j.ijrobp.2004.07.666

[14] BALLARE A, SALEV MD, LOI G, FERRARI G, BELDI D et al. Conformal radiotherapy of clinically localized prostate cancer: analysis of rectal and urinary toxicity and correlation with dose-volume parameters. Tumori. 2009; 95: 160-168.

[15] GULliford SL, KERWYN F, MORGAN RC et al. Dose-volume constraints to reduce rectal side effects from prostate radiotherapy: evidence from MRC RT01 trial ISRCTN 47772397. Int. J. Radiation Oncology Biol. Phys. 2010; 76: 747-754. http://dx.doi.org/10.1016/j.ijrobp.2009.02.025

[16] COX JD, STETZ J, PAJAK TF. Toxicity criteria of the Radiation Therapy Oncology Group (RTOG) and the European Organization for Research and Treatment of Cabcer (EORTC). Int. J. Radiation Oncology Biol. Phys. 1995; 31: 1341-1346. http://dx.doi.org/10.1016/0360-3016(95)00060-C

[17] VISWANATHAN AN, YORKE ED, MARKS LB et al. Radiation dose/volume effects of the urinary bladder. Int. J. Radiation Oncology Biol. Phys. 2010; 76: S116-S122. http:// dx.doi.org/10.1016/j.ijrobp.2009.02.090

[18] MARZI S, SARACINO B, PETRONGARI MG, ARCANGELI S, GOMELLINI $S$ et al. Modeling of $\alpha / \beta$ for late rectal toxicity form a randomized phase II study: conventional versus hypofractionated scheme for localized prostate can- 
cer. J. Exp. Clin. Cancer Res. 2009; 28: 117. http://dx.doi. org/10.1186/1756-9966-28-117

[19] RANCATI T, FIORINO C, GAGLIARDI G, CATTANEO GM, SANGUINETI G et al. Fitting late rectal bleeding data using different NTCP models: result from an Italian multi- centric study (AIROPROS0101). Radiotherapy an Oncology. 2004; 73: 21-32. http://dx.doi.org/10.1016/j. radonc.2004.08.013

[20] LUIJK P VAN, DELVIGNE TC, SCHILSTRA C, SCHIPPERS JM. Estimation of parameters of dose-volume models and their confidence limits. Phys. Med. Biol. 2003; 48: 1863-1884. http://dx.doi.org/10.1088/0031-9155/48/13/301
[21] LYMAN JT. Complication probability as assessed from dose volume histograms. Rad. Res. 1985; 104: S13-S19. http:// dx.doi.org/10.2307/3576626

[22] FOWLER JF. The linear-quadratic formula and progress in fractionated radiotherapy. Br. J. Radiol. 1989; 62: 679-694. http://dx.doi.org/10.1259/0007-1285-62-740-679

[23] KUTCHER GJ, BURMAN C, BREWSTER L, GOITEIN M, MOHAN R. Histogram reduction method for calculating complication probabilities for three-dimensional treatment planning evaluations. Int. J. Radiation Oncology Biol. Phys. 1991; 21: 137-146. http://dx.doi.org/10.1016/0360-3016(91)90173-2 